\title{
IL TERREMOTO JONICO DEL 22 APRILE 1948
}

\author{
Paolo Emilio Valle
}

Allo scopo di contribuire alla localizzazione dei centri sismici che interessano l'Italia e per essere in possesso degli elementi fondamentali necessari ad una successiva ricerca sulla costituzione della crosta terrestre sotto il Mediterraneo, sono state calcolate le coordinate epicentrali e il tempo origine del terremoto del 22 aprile 1948. La scossa ha avuto effetti distruttivi nella regione dell'Isola di Leucade, ¿̀ stata avvertita a Prveza, Agrinion, Zante. Missolonghi, Corfù ed ha carattere superficiale.

Da una stima eseguita mediante i sismogrammi delle stazioni di Roma, Bologna e Salo, la magnitudo (1) è risultata $634^{*}$. In relazione a questo valore della magnitudo, l'energia liberata all'ipocentro si può valutare a circa $10^{23.5}$ erg e l'intensita all'epicentro corrispondente al $9^{\prime \prime}$ grado della scala Mercalli modificata.

Una prima localizzazione dell'epicentro è stata ottenuta col metodo della proiezione stereografica, applicato ad una decina di stazioni, che ha fornito il seguente risultato:

$$
\begin{array}{ll}
\lambda=20^{\circ}, 5 & \mathrm{E} \\
\varphi=38^{\circ}, 5 & \mathrm{~N} \text { (geografica) } \\
\varphi^{\prime}=38^{\circ}, 312 & \mathrm{~N} \quad \text { (geocentrica) }
\end{array}
$$

In base alle coordinate epicentrali provvisorie [1], il tempo origine medio, dedotto dai tempi di arrivo delle onde $P$, letti direttamente nei sismogrammi o copie degli osservatori elencati nella tal,ella I, e dai tempi di tragitto delle analoghe onde, calcolati da Jeffreys $\left({ }^{2}\right)$ per centri di perturbazione superficiali, vale

$$
H=10^{\mathrm{h}} 42^{\mathrm{m}} 40^{\mathrm{s}, 8} \quad \text { (T.M.G.) }
$$

I valori [1] e [2] sono stati presi come valori di partenza per l'applicazione del metodo delle approssimazioni successive $\left({ }^{3}\right)$.

Se si indica con $f$ il tempo di tragritto delle onde $P$, con $\gamma_{0}, \varphi_{0}^{\prime}$ le

(*) Il Prof. Gutenberg mi ha gentilmente comunicato che l'analoga stima eseguita a Pasadena ha dato lo stesso valore. 
coordinate geocentriche dell'epicentro, con $H_{0}$ il tempo origine, con $l_{\mathrm{i}}$ le differenze tra $\mathrm{i}$ tempi di arrivo calcolati mediante $\mathrm{i}$ valori di partenza e i tempi osservati, si ha per ogni Stazione

$$
l_{\mathrm{i}}+\frac{\partial f}{\partial \lambda_{\mathrm{i}}} \varepsilon+\frac{\partial f}{\partial \varphi_{i}^{\prime}} \eta+\tau=0
$$

dove si è posto

$$
(i-1,2, \ldots, n)
$$

$$
\begin{aligned}
& \varepsilon=\lambda_{0}-\lambda \\
& \eta=\uparrow_{0}^{\prime}-\varphi^{\prime} \\
& \tau=H_{0}-H
\end{aligned}
$$

e nell'ipotesi che $\varepsilon$, $\eta$ e $\tau$ siano sufficientemente piccoli in modo da poter trascurare i loro prodotti e i loro quadrati. $n$ indica il numero delle Stazioni.

Le derivate $\partial f / \partial \lambda_{i}$ e $\partial f / \partial \varphi_{i}^{\prime}$ si possono calcolare tenendo presente che si può scrivere

$$
\frac{\partial f}{\partial \lambda_{i}}=\frac{\partial f}{\partial \Delta_{i}} \frac{\partial \Delta_{i}}{\partial \lambda_{i}} \quad, \quad \frac{\partial f}{\partial \varphi_{i}^{\prime}}=\frac{\partial f}{\partial \Delta_{i}} \frac{\partial \Delta_{i}}{\partial \varphi_{i}^{\prime}}
$$

La $\partial f / \partial \Delta_{\mathrm{i}}$ ¿ stata ottenuta dai tempi di tragitto calcolati da Jeflreys e le altre due derivate dalle espressioni

$$
\begin{aligned}
& \frac{\partial \Delta}{\partial \lambda_{i}}=\frac{\cos \varphi^{\prime}\left(a_{i} \sin \lambda_{-}-b_{1} \cos \lambda_{1}\right)}{\sin \Delta_{i}} \\
& \frac{\partial \Delta}{\partial \varphi_{i}^{\prime}}=\frac{\sin \varphi^{\prime}\left(a_{i} \cos \lambda+b_{i} \sin \lambda\right)-c_{1} \cos \varphi^{\prime}}{\sin \Delta_{i}}
\end{aligned}
$$

nelle quali $a_{i}, b_{i}, c_{\mathrm{i}}$ sono $\mathrm{i}$ coseni direttori della $\boldsymbol{t}^{\mathrm{mn}}$ Stazione.

Dato che le [6] non sono indipendenti, ma legate dalla relazione

$$
\left(\frac{1}{\cos \varphi^{\prime}} \frac{\partial \Delta}{\partial \lambda_{i}}\right)^{2}+\left(\frac{\partial \Delta}{\partial \varphi_{i}}\right)^{2}=1
$$

la verifica dei calcoli può essere fatta agevolmente.

La tabella I contiene i valori dei coefficienti e dei termini noti del sistema di equazioni [3], che sono stati usati per il calcolo.

I ralori approssimati più probabili delle incognnite e $\mathrm{i}$ rispettivi errori medi sono

$$
\begin{aligned}
& \varepsilon=-0^{\circ}, 02+0^{\circ}, 04 \\
& \eta=-0^{\circ}, 01-0^{\circ}, 04 \\
& \tau=0^{\circ} ; 0 \pm 0^{\circ}, 4
\end{aligned}
$$


Tabrela I

\begin{tabular}{|c|c|c|c|c|}
\hline N. & Stazione & $\frac{\partial f}{\partial \lambda_{i}}$ & $\frac{\partial f}{\partial \varphi_{i}^{\prime}}$ & $\begin{array}{l}l_{1} \\
\text { sec. }\end{array}$ \\
\hline $\begin{array}{r}1 \\
2 \\
3 \\
1 \\
5 \\
6 \\
7 \\
8 \\
9 \\
10 \\
11 \\
12 \\
13 \\
14 \\
15 \\
16 \\
17 \\
18 \\
19 \\
20 \\
21\end{array}$ & $\begin{array}{l}\text { Sofia } \\
\text { Roma } \\
\text { Bucarest } \\
\text { Trieste } \\
\text { Budapest } \\
\text { Graz } \\
\text { Praga } \\
\text { Helwan } \\
\text { Zurigo } \\
\text { Basilea } \\
\text { Strasburgo } \\
\text { Jena } \\
\text { Gottingar } \\
\text { Tortosa } \\
\text { Parigi } \\
\text { Uccle } \\
\text { I.und } \\
\text { Copenhagen } \\
\text { Toledo } \\
\text { Lisbona } \\
\text { Bergen }\end{array}$ & $\begin{array}{l}-4,9 \\
+9,4 \\
+6,1 \\
+5,9 \\
+1,2 \\
+4,0 \\
+3,3 \\
-8,0 \\
+6,9 \\
+7,0 \\
+6,4 \\
+4,2 \\
+1,3 \\
+9,8 \\
+7,2 \\
+6,1 \\
+2,3 \\
+2,5 \\
+8,9 \\
+7,6 \\
+2,4\end{array}$ & $\begin{array}{l}-12,7 \\
-\quad 7,3 \\
-11,7 \\
-11.6 \\
-13,7 \\
-12,8 \\
-12,7 \\
+\quad 8,7 \\
-10,2 \\
-9,3 \\
-10,1 \\
-12,1 \\
-11.7 \\
-3,3 \\
-8,9 \\
-10,0 \\
-12,1 \\
-12,0 \\
-2.4 \\
-1,7 \\
-\quad 9,2\end{array}$ & $\begin{array}{l}-0,7 \\
-0,7 \\
-1,0 \\
+1,1 \\
-0,7 \\
+0,6 \\
+0,8 \\
+0,4 \\
+0,9 \\
+1,1 \\
+0,7 \\
-0,1 \\
-0,2 \\
-0,9 \\
-1,0 \\
-1,1 \\
+0,1 \\
+1,2 \\
+0,1 \\
+1,0 \\
-0,9\end{array}$ \\
\hline
\end{tabular}

\section{TABELLA II}

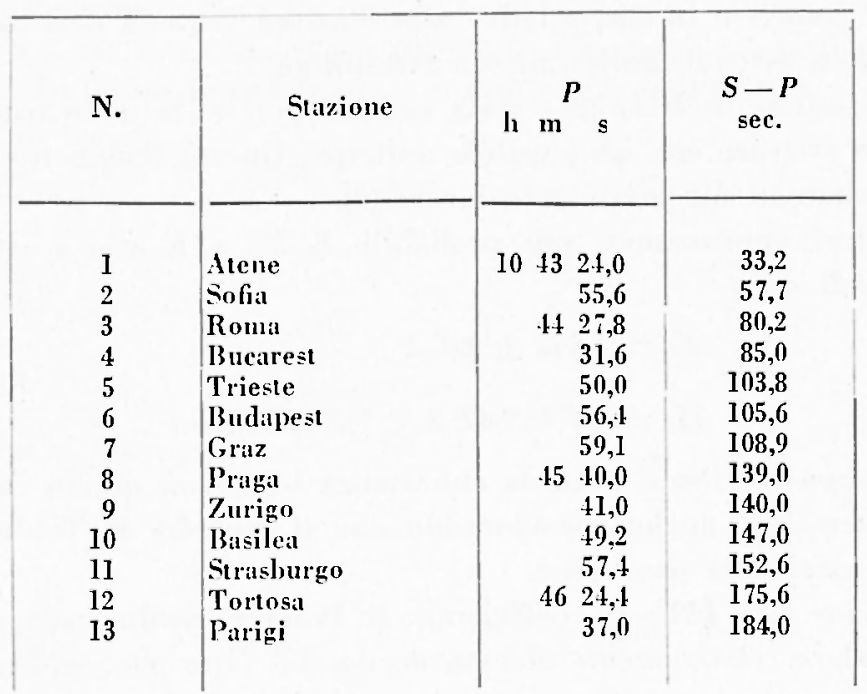


Questi valori delle correzioni da apportare ai dati di partenza sono sufficientemente piccoli e si rende quindi superflua un'ulteriore approssimazione. Pertanto le coordinate definitive dell'epicentro e il tempo origine risultano

$$
\begin{aligned}
& \lambda_{0}=20^{\circ \prime}, 48-0^{\circ}, 04 \quad \mathrm{E} \\
& \tau_{0}=38^{\prime \prime}, 49-0^{\circ}, 04 \quad \mathrm{~N} \text { (geografica) } \\
& H_{0}=10^{\mathrm{h}}+2^{\mathrm{m}}+40^{\circ}, 80^{\circ}, 0^{\circ}, 4 \text { (T.M.G.) }
\end{aligned}
$$

Si è gia accennato al fatto che le registrazioni indicano che il centro di perturbazione $\dot{e}$ superficiale, tuttavia $\grave{e}$ sembrata opportuna una verifica del tempo origine con altro metodo indipendente dai precedenti.

A tale scopo è stato usato il metodo di Wadati (\%), il quale si serve, come ̀̀ noto, dei tempi di arrivo delle onde $P$ e delle differenze $S-P$.

Se le stazioni non sono troppo lontane dal centro di perturbazione, risulta approssimativamente

$$
\frac{S-P}{P-H_{0}}=\frac{1}{K}=\text { costante }
$$

La costante è legata al coefficiente di Poisson dalla relazione

$$
\sigma=0,5-0,5 \frac{K^{2}}{1+2 K}
$$

La tabella II contiene i tempi di arrivo delle onde $P$ e le differenze $S-P$ espresse in sec, relative alle Stazioni usate. I dati di Atene sono stati presi dal Bollettino di Strasburgo.

La Stazicne di Helwan è stata esclusa, perché le onde trasversali sembrano arrivare con un sensibile anticipo. Questo fenomeno è stato già segnalato in un precedente lavoro (").

I valori approssimati più probabili di $H_{0}$ e $K$ con i rispettivi errori medi sono

$$
\begin{aligned}
& K=1,272 \\
& H_{0}=10^{\mathrm{h}} 42^{\mathrm{m}} 42^{\mathrm{s}}, 3,012 \\
& 1^{\mathrm{s}}, 5 \quad \text { (T.M.G.) }
\end{aligned}
$$

Il tempo origine si accorda abbastanza bene con quello calcolato precedentemente, anche considerando che il metodo di Wadati non consente un'elevata precisione.

In base alla [11] il coefficiente di Poisson risulta pari a 0,27. Questo valore relativamente elevato deriva dal fatto che, sebbene non 
siano stati inclusi nel calcolo i dati delle Stazioni eccessivamente distanti dal centro di perturbazione, anche quelle prese in considera. zione sono un po' troppo lontane e quindi l'onda si c̀ propagata fino ad una profondita piuttosto elevata.

$$
\text { Roma - Istituto Nazionale di Geofisica -- Gennaio } 1951
$$

\section{RIASSUNTO}

Allo scopo di contribuire alla localizazione dei centri sismici che interessatio l'Italia o per essere in possesso dei dati fondamentali per una successiva ricerca, sono state calcolate le coordinate epicentrali e il tempo origine del terremoto Jonico del 22 aprile 1948.

\section{BIBI.IOGRAFIA}

(1) Guteviring B. and F. C. Richtr.r: Earthquale Magnitude, Intensily, Energy and Acceleration - Bull. Seism. Soc. of Am. XXXII. 163-191 (1942).

- Dı Fituppo D. e L. Marceldr: Magnitudo ed energia dei terremoti in Italia . Anuali di Geofisica II, 337.3 .18 (1950).

(-) Jefrneys HL: The Time of $P, S$ and $S K S$, and the velocities of $P$ and $S$. Geopl. Suppl. IT, 498-533 (1939).

(3) Gruck: L.: Herdbestimmung bei Erdbeben nus den Ankunftszeiten - Naclıichten der Koniglichen Gesellschaft der Wissenselazten zu Gottingen, Matlo-pliys. Klisse, 331-319 (1910).

- Valf. P. E.: Sulla determinazione delle coordinate epicentrali di un sisma lontamo - Boll. Soc. Sism. Italiana XL, 73-81 (19)12).

(4) Wabarı K.: On the Travel Time of Earihquake uares. Part II - Geoph. Mag. 7. 101-111 (1933).

(i) VAlite P. E.: Contributo allo studio dolle caratteristiche sismiche del Mediterraneo Centro-Orientale - Annali di Geofisica: I, 266-278 (19.48). 